\title{
Rotary motion and efferent readiness ${ }^{1}$
}

CLARKE A. BURNHAM ${ }^{2}$ AND D. ROWLAND AERTKER, JR. UNIVERSITY OF TEXAS, AUSTIN

Two experiments were conducted to test the influence of a readiness to make a rotary movement on the perception of rotary mation. In both experiments, Os monocularly viewed a stimulus whose direction of rotation is ambiguous while they, were set or prepared to make a crank-turning motor response in a particular direction. Experiment 1 demonstrated that the initially perceived direction of rotation was more stable, i.e., lasted longer, if it was consistent with the direction in which Os were prepared to turn the crank. The effect of a readiness for motor activity on the stability of rotary motion was similar to the previously determined effect of overt motor activity. Experiment 2 demonstrated that the perception of the initial direction of rotation was shaped by a readiness to make a directional motor response.

What is the relationship between visual perception and motor activity? There is experimental support for the proposition that perception is influenced by the actions of the O. Burnham (1969) demonstrated that the time to apparent reversal of rotation of a stimulus whose direction of rotation is ambiguous was longer when the initially perceived direction of rotation was consistent with the motor activity of an $O$. Os simultaneously turned a crank and viewed an object capable of apparent reversal of rotation. If the perceived direction was consistent with the turning direction, the perception was more stable than when the perceived direction and the motor movements were inconsistent. Shopland and Gregory (1964) found that apparent reversals of orientation of a wire-frame cube were reduced when the OS simultaneously touched and viewed the cube. Another source of evidence for a linkage between perception and motor activity is the relationship between eye movements and the Müller-Lyer illusion. Several early investigators found that saccadic eye movements overshoot the perceptually long side of the illusion figure and undershoot the perceptually short side. Festinger, White, and Allyn (1968) confirmed these findings and also reported that a decrease in the magnitude of the illusion is associated with a correction in the saccadic eye movements made while viewing the illusion.

Yet it is absolutely clear that visual perception can occur in the absence of overt motor activity. Contour, shape, and length can be accurately perceived when stimuli are exposed too rapidly for eye movements or other gross motor movements to occur. A motor theory of perception, if phrased in terms of overt motor activity, is unsatisfactory. There is, however, experimental evidence to support the hypothesis that perception is determined by the readiness to respond activated by the afferent visual stimulation. Such an efferent readiness theory of perception has been proposed by Festinger, Burnham, Ono, and Bamber (1967). It has been supported by several studies of adaptation to the curvature induced by wearing prism spectacles (Festinger et al, 1967; Burnham, 1968a). Those Os in experimental conditions designed to require the learning of new afferent-efferent associations adapted to the prism-induced curvature. Efference theory has also been supported by a study of the decrement in the Müller-Lyer illusion (Burnham, 1968b). Os who were given an opportunity to learn new saccadic eye movements to the illusion figure showed a decrement in the magnitude of the illusion. As the efferent programs for saccadic eye movements become recalibrated, the illusion decreases (Festinger et al, 1968; Burnham, 1968b).

The experiments reported in this study were designed to demonstrate that a readiness to make rotary movements of the arm and hand affects the perception of rotary motion of an ambiguous stimulus. Experiment 1 is an extension of the consistency studies (Burnham, 1969) that demonstrated that efference issued concurrently with a perceived direction of rotation influences the stability of the perceived rotary movement. The hypothesis tested in Experiment 1 of the present investigation is that a readiness to engage in a motor activity will influence the stability of rotary movement. If a person is prepared to turn a crank clockwise (CW), his perception of $\mathrm{CW}$ rotation will be more stable than his perception of counterclockwise (CCW) rotation. Experiment 2 was designed to demonstrate that the initial direction of rotation of an ambiguous object can be shaped by a readiness to issue efference to the arm and hand. Specifically, it was hypothesized that a person ready to tum a crank $\mathrm{CW}$ would tend to see $\mathrm{CW}$ rotation rather than $\mathrm{CCW}$ rotation.

\section{EXPERIMENT 1}

The $O s$ in Experiment 1 observed a rotating ellipse. The initially perceived direction of rotation of the ellipse was controlled on each experimental trial by allowing binocular viewing of the rotating ellipse prior to monocular viewing. When a rotating ellipse is viewed monocularly under appropriate stimulus conditions, alterations in its perceived movement occur (Day \& Power, 1965; Hershberger, 1967). These alterations include reversals of direction, oscillation, an apparent expansion and contraction of the sides of the ellipse, and a flapping of the sides about the axis of rotation. During the experimental trials, the Os were told to signal the reversal or other atteration in perceived movement by turning a crank in a specified direction. This manipulation was, of course, designed to activate efferent readiness while the rotating ellipse was being viewed. It was expected that the initially perceived direction of rotation would be more stable if it was consistent with the rotary movement that the Os were prepared to perform.

\section{Apparatus}

The apparatus used in this experiment was similar to that described in the consistency studies (Burnham, 1969). The $O$ sat at one end of a wooden frame, with his chin in a padded support to minimize head movements. The ellipse was located approximately 55 in. from $O$ 's eyes near the other end of the frame. It had a major axis of 3 in., a minor axis of $3 / 4$ in., was cut from .029-in. sheet metal, and was painted flat black. The ellipse was rotated about its minor axis by a reversible motor at the rate of $60 \mathrm{rpm}$. The crank was located in front of $O$, and he turned it with his right hand. The center of rotation of the crank and the ellipse were aligned with $O$ 's right eye, and the center of the ellipse was at eye level. The ellipse was viewed through 2 in. square apertures located 1 in. in front of the $O$ 's eyes. Shields were used to control $O$ 's view of the ellipse through these apertures.

\section{Procedure}

Each experimental session consisted of a series of binocular test trials, some practice or familiarization trials, and the 
experimental trials. The binocular test trials insured that each experimental 0 could veridically perceive the direction in which the ellipse was rotating while viewing it binocularly. These trials consisted of a series of $1.5 \mathrm{sec}$ exposures of the rotating ellipse. After each exporure, 0 called out the preceived direction of rotation, $\mathrm{CW}$ or $\mathrm{CCW}$. The experimental session was terminated for those Os who were unable to make eight successively correct reports within 16 such trials.

At the beginning of each practice and experimental trial, $O$ 's view of the ellipse was blocked. After starting the ellipse, E depressed a switch that caused the first shield to drop and allowed $\mathrm{O}$ to view the rotating eflipse binocularly for $1 \mathrm{sec}$. A second shield then dropped, blocking $O$ 's left-eye view of the ellipse. At this time, a stopclock was started to time the stability of rotary motion. $O$ was instructed to report the monocularly perceived direction of rotation as soon as possible after the second shield dropped. Turning the crank stopped the timing clock and also caused a third shield to drop, which again blocked $O$ 's view of the ellipse and ended the trial. During the four practice trials, $O$ verbally reported reversals of rotation and $E$ turned the crank. These trials were designed to familiarize $O$ with the perception of reversal and to remove the initially long and variable latencies to reversal, which typically occur on the first monocular trials, from the experimental data. The instructions to $\mathbf{O}$ were: "While you are seeing the ellipse monocularly, it will reverse the direction in which it is going. If going $\mathrm{CW}$, it will start going $\mathrm{CCW}$, and if going $\mathrm{CCW}$, it will start going $\mathrm{CW}$.... All I want you to do is to tell me when the reversal of direction occurs. Just say, 'It reversed." "

The eight experimental trials followed these practice trials. In the experimental trials, $O$ was told to signal the reversal by turning the crank around once when he saw the reversal. $O$ was told that $E$ was measuring his reaction time (RT) from the onset of reversal to the crank-turning signal of reversal and that he should be "... all set to turn the crank as soon as [he saw] that change in direction."

One-half of the Os were told to signal reversals by tuming the crank CW and one-half were instructed to turn the crank CCW. On half the trials, the actual direction of rotation was $C W$, and on the other half, it was CCW. The order of rotation for the experimental trials was BAABABBA. For one-half of the $O s$ in each crank-turning group, $A$ indicates $C W$ rotation, and for the other half, $A$ indicates $\mathrm{CCW}$ rotation.

\section{Observers}

The Os were introductory psychology students fulfilling a portion of the course requirements. They were assigned randomly to the experimental conditions until there were 20 usable Os. Three Os failed the binocular test. The data from one other $O$ were not analyzed since he did not see the actual direction of rotation on three of the eight experimental trials.

\section{Results and Discussion}

The experimental hypotheses were tested by comparing the times to reversal for each $O$ on those trials when the initial direction of rotation was consistent with the direction in which he was prepared to turn the crank with the times to reversal on those trials when the initial direction and readiness were inconsistent. Although the perceived direction of rotation was experimentally manipulated, there were seven Os who reported rotation in a direction opposite to the veridical direction on one of the eight trials. This may have resulted from misperception of the direction during binocular viewing or from a change in apparent direction between the binocular and monocular viewing. The direction of rotation on these trials was considered to be the direction that $O$ reported seeing. Thus, these seven Os had either five consistent and three inconsistent trials $(N=4)$ or three consistent and five inconsistent trials $(\mathrm{N}=3)$. When the readiness and perception were consistent, the times to reversal were approximately $1 \mathrm{sec}$ longer than when they were inconsistent. The mean time to reversal for the consistent trials was $6.73 \mathrm{sec}$; for the inconsistent trials, it was $5.74 \mathrm{sec}$. The difference between these times is statistically significant $(t=2.13$, df $=19$, $\mathrm{p}<.05$ ).

The magnitude of the consistency effect produced by efferent readiness is similar to that produced by overt motor activity. The times to apparent reversal of an ellipse reported by Burnham (1969) are $7.68 \mathrm{sec}$ when the $O$ was tuming the crank in a direction consistent with the perception and $6.78 \mathrm{sec}$ when the motor movements and the perception were inconsistent. Readiness for motor activity and actual motor activity have similar effects on the stability of perceived rotary motion.

\section{EXPERIMENT 2}

If perception is shaped by efferent readiness, then the perception of rotary motion should correspond to the stimulus-related movements one is prepared to make at the onset of visual stimulation. This hypothesis was tested in Experiment 2. Two groups of Os were run in this study. The experimental condition was designed to test the effect of efferent readiness on rotary motion. The control condition was designed to insure that a readiness to respond, rather than other variables, was responsible for the observed data.

The Os in both the experimental or readiness condition and the control or delayed-readiness condition were told to turn a crank in a specified direction to the sound of a buzzer. All Os first saw the rotating stimulus monocularly, then made their crank-turning response to the buzzer, and then signaled the perceived direction of rotary motion of the stimulus by turning the crank in the perceived direction of rotation. The $O s$ in the readiness condition did not know whether the buzzer would sound almost immediately after they first saw the stimulus or $2-3 \mathrm{sec}$ after the onset of the stimulus. Thus, they had to be prepared to issue efference at the beginning of each trial, at the onset of the afferent visual stimulation from the rotating object. The buzzer always sounded $2.3 \mathrm{sec}$ after the onset of the stimulus for the $O s$ in the delayed-readiness condition; they did not have to be prepared to issue efference to the arm and hand at the beginning of the trial, and hence it was not expected that their perception of rotation would be influenced by the readiness to respond to the buzzer.

\section{Apparatus}

The same basic apparatus was used in this experiment as in Experiment 1. A wire-frame truncated pyramid was used as the stimulus rather than the ellipse to minimize apparent reversals of rotary motion during the experimental trials, which lasted approximately $6 \mathrm{sec}$. Time to reversal for the pyramid tends to be longer than for the ellipse. Also, pretest Os reported that it was easier to perceive unequivocally a particular direction of rotation with the pyramid than with the ellipse. The pyramid was constructed from $3 / 32$-in. rods and painted flat black. The larger base was a 3 -in. square, the smaller base, a 1-1/8-in. square. The altitude was $21 / 4 \mathrm{in}$. The pyramid was rotated at $60 \mathrm{rpm}$ by a reversible motor. It was oriented so that its bases were perpendicular to the plane of rotation. The perception of a particular direction of rotation of such a stimulus depends on the perceived orientation of its bases, which was ambiguous under the monocular viewing conditions of this study. A buzzer served as the stimulus for rotation of the crank. Turning the crank stopped the buzzer and also stopped a clock that was ostensibly used to measure RT to the buzzer. 


\section{Procedure}

All Os first engaged in a period of tracking the rotating pyramid with the crank. This tracking session was designed to link turning the crank with the rotation of the pyramid and to assure that Os saw the pyramid rotate both $\mathrm{CW}$ and $\mathrm{CCW}$. The pyramid was viewed monocularly. Os were told to turn the crank in the same direction as the pyramid was turning and to reverse the direction of crank turning when the pyramid reversed directions. E reversed the actual direction of rotary motion if no spontaneous reversals occurred within the first two 15-sec periods of this tracking session and thereafter if no spontaneous reversals occurred within 30 -sec periods. The tracking session continued until $O$ saw four spontaneous reversals.

The $O s$ in the readiness condition were then told that the purpose of the experiment was to compare their RT to a buzzer on two types of trials. They were told that on some trials, the decision trials, they were to respond to the buzzer by turning the crank around once in a previously specified direction after having engaged in a "simple decision-making task," which was to determine the direction of rotation of the pyramid. On other trials, the no-decision trials, they were told that their response would be a measure of "raw reaction time," since the buzzer would sound as soon as they first saw the pyramid and they would not have time to decide the direction of rotation. The Os did not know whether a trial would be a decision or a no-decision trial; the difference between the trials was that the buzzer sounded 2-3 sec after Os saw the rotating pyramid on the decision trials, and it sounded as soon as they saw the pyramid on the no-decision trials. The experimental independent variable was manipulated by telling $\mathrm{O}$ prior to each trial whether to turn the crank $\mathrm{CW}$ or $\mathrm{CCW}$ when he heard the buzzer. At the beginning of each trial, $O$ 's view of the pyramid was blocked by a shield. E first stated the direction of rotation for the $\mathrm{RT}$ response. $\mathrm{E}$ then started the pyramid and shortly thereafter dropped the shield, allowing $O$ to see the pyramid with his right eye. This procedure insured that the pyramid would be seen initially in different orientations over the trials. After responding to the buzzer, $O$ turned the crank to correspond to the perceived direction of rotation, which provided $E$ with the measure of his perceived direction of rotation. After $O$ tracked the pyramid for $3-4$ rotations, E ended the trial by stopping the pyramid and replacing the shield.

There were 16 decision trials and eight no-decision trials arranged randomly, with the restriction that there be two no-decision trials in each block of six trials. This restriction was imposed to insure that $O$ was prepared to turn the crank at the time the shield dropped on every trial. The actual direction of rotation of the pyramid and the specified direction of crank turning were randomized, with the restriction that four $\mathrm{CW}$ and four $\mathrm{CCW}$ trials appear in each block of eight trials and that there be two trials of the four possible combinations of direction of rotation and crank-turning direction in each block of eight trials. The order of presentation of the 24 trials was reversed for one-half of the Os. Only the responses to the decision trials were used to test the effect of efferent readiness on the perception of rotary motion.

The procedure for the delayed-readiness group was similar to that for the readiness group except for the elimination of the no-decision trials. The Os were told that the buzzer would sound $2-3 \mathrm{sec}$ after they saw the rotating pyramid and that $E$ was interested in their RT after having made a decision. Thus, the delayed-readiness Os did not have to be prepared to respond when they first saw the pyramid. These Os also participated in 24 trials. The consistency effect was analyzed only for those 16 trials that corresponded to the 16 decision trials for the readiness group.

\section{Observers}

The Os were 33 undergraduate students fulfilling an introductory psychology course requirement. The first 17 Os were assigned to the experimental group. The data from one $O$ were not analyzed since he was unable to signal a direction of rotation immediately after the $R T$ response due to a rapid onset of the perception of oscillation. The next 16 Os were assigned to the delayed-readiness, or control, group.

\section{Results and Discussion}

Each of the 16 decision trials was scored as consistent if the perceived direction corresponded to the direction in which $\mathbf{O}$ was prepared to turn the crank and as inconsistent if the directions differed. There were two Os in the experimental group whose responses on one trial were not scored since they indicated that reversals or oscillation occurred between the initial perception and the time to signal the perceived direction of rotation. The mean difference between the number of consistent and inconsistent trials for the readiness-condition $\mathrm{Os}$ was 1.625 . The corresponding difference for the control-group Os was -.125 . The consistency effect for the experimental group is significantly different from a null hypothesis value of zero $(t=3.99, \mathrm{df}=15$, $\mathrm{p}<.01)$. That for the control group does not differ from zero $(t=-.20)$. The consistency effect for the experimental group differs from that of the control group at a level approaching statistical significance $(t=1.98, d f=30, p<.10)$. In sum, it is apparent that a readiness to perform a directional motor response tends to result in a perception consistent with that readiness.

Although the consistency effect is significant, an examination of the data for each $O$ over the experimental trials suggests that the effect of readiness on perceived direction was reduced by a tendency for $\mathrm{Os}$ to see the pyramid rotate in the same direction, usually $\mathrm{CW}$, on most of the trials. The $O s$ in the readiness group saw the pyramid rotate in the same direction on $92 \%$ of the decision trials and on $96 \%$ of the no-decision trials. The control-group Os saw it rotate in the same direction on $82 \%$ of the 24 trials. This perseveration effect was significantly more pronounced for the readiness Os. A contingency classification of the groups and the number of dominant responses on the 16 decision trials, split at 13 or more vs less than 13 , resulted in $\chi^{2}=5.57, \mathrm{df}=1, \mathrm{p}<.02$. It is possible that the perseveration effect resulted from the requirement for rapid perception of direction of rotary motion. This requirement was probably stronger in the readiness condition since these Os had to see some direction of rotation rapidly on the no-decision trials. Because the stimulus was similar on all trials, the requirement for rapid perception could be met by seeing only one direction of rotation. When the perseveration effect was broken, the experimental Os almost always saw the pyramid rotate in the direction in which they had been prepared to turn the crank. When the control-group Os saw the pyramid rotate in the least-preferred direction, there was no relationship between the perception and the crank-turning direction.

The consistency effect was not reduced by a tendency for Os to perceive the actual direction of rotation. The $O s$ in the readiness group perceived the actual direction of rotation on $49 \%$ of the decision trials and on $51 \%$ of the no-decision trials. They were also accurate on an average of $49 \%$ of those decision trials in which the perceived direction of rotation corresponded to the direction in which they were prepared to turn the crank. The Os in the delayed-readiness group were accurate on $51 \%$ of all trials.

The consistency effect was apparently not caused by a tendency to report movement consistent with the specified turning direction produced by experimental demands or ease of motor activity. The readiness Os were not 
responding to a demand or expectancy to see the pyramid rotate in the same direction as they had been told to turn the crank, for the consistency effect was not observed in the delayed-readiness group, which was subjected to the same demands. It might have been easier to report movement consistent with the specified turning direction since $O$ could then continue to turn the crank in the same direction. However, this did not occur in the delayed-readiness group. The consistency effect did not stem from heightened visual acuity, for it was independent of perceptual accuracy. It is probable that the consistency effect was reduced by the perseveration phenomenon. In these experimental conditions, Os tend to see the same direction of rotary motion on all experimental trials.

\section{CONCLUSION}

These two experiments demonstrate that efferent readiness influences the perceived direction of rotation of ambiguous stimuli. Experiment 1 showed that a readiness to respond has the same effect on the stability of rotary motion as overt motor activity. Experiment 2 showed that a readiness to respond at the onset of visual stimulation shapes the perception of rotary motion. Both experiments support the theory that what one sees is determined by how one is prepared to respond.

\section{REFERENCES}

BURNHAM, C. A. Adaptation to prismatically induced curvature with nonvisible arm movements. Psychonomic Science, 1968a, 10, 273-274.

BURNHAM, C. A. Decrement of the Müller-Lyer illusion with saccadic and tracking eye movements. Perception \& Psychophysics, $1968 \mathrm{~b}, 3,424-426$.

BURNHAM, C. A. Consistency between motor activity and perceived direction of rotation. Perception \& Psychophysics, 1969, 5, 29-32. DAY, R. H., \& POWER, R. P. Apparent reversal (oscillation) of rotary motion in depth: An investigation and a general theory. Psychological Review, 1965, 72, 117-127.

FESTINGER, L., BURNHAM, C. A., ONO, H., \& BAMBER, D. Efference and the conscious experience of perception. Journal of Experimental Psychology, Monograph Supplement, 1967, 74(4, Whole No. 637).

FESTINGER, R. L., WHITE, C. W., \& ALLYN, $M$. R. Eye movements and decrement in the Müller-Lyer illusion. Perception \& Psychophysics, 1968, 3, 376-383.

HERSHBERGER, W. A. Comment on "Apparent reversal (oscillation) of rotary motion in depth." Psychological Review, 1967, 74, 235-238.

SHOPLAND, C. D., \& GREGORY, R. L. The effect of touch on a visually ambiguous three-dimensional figure. Quarterly Journal of Experimental Psychology, 1964, 16, 66-70.

\section{NOTES}

1. Robert Sweeney conducted Experiment 1. Rowland Aertker had primary responsibility for Experiment 2.

2. Address: Department of Psychology, Mezes Hall, University of Texas, Austin, Texas 78712 .

(Accepted for publication July 21, 1969.) 